\title{
Modeling and Simulation Technology of High Frequency Vibratory Stress Relief Treatment for Complex Thin - Walled Workpiece
}

\author{
Xian-yang ZHAO ${ }^{1}$, Nian-song ZHANG $^{1}$ and Ai-min WANG ${ }^{2}$ \\ ${ }^{1}$ School of Mechanical Engineering, Nanjing University of Science and Technology, Nanjing 210000, China \\ ${ }^{2}$ School of Mechanical Engineering, Beijing Institute of Technology, Beijing 100081, China
}

\begin{abstract}
The current research based on vibratory stress relief (VSR) is focused on low frequency excitation, but it is seldom mentioned for complex thin-walled workpiece with higher natural frequency. In this paper, the mechanism of high frequency VSR is studied by microdynamic theory. Aiming at the rotating frame of complex thin-walled workpiece in aerospace equipment, the high frequency VSR numerical simulation is carried out by finite element software Abaqus, the modal analysis technology and harmonic response analysis technology are proposed, and the exciting force, excitation frequency and other important parameters are determined. The simulation results are analyzed to verify the superiority of high frequency VSR to eliminate residual stresses of complex thin-walled workpieces, and provide a theoretical basis for optimal selection of excitation parameters for high frequency VSR.
\end{abstract}

\section{Introduction}

Complex thin-wall workpiece has the characteristics of light weight, high strength and so on, and is widely used in aerospace and military industry. In the process of machining, it is easy to produce uneven plastic deformation, resulting in residual stress, the existence of residual stress is the main cause for the cracking or deformation of the workpiece. According to the literature [1], compared with the traditional way of eliminating residual stress such as natural aging and thermal aging, in twenty-first century, the technology of VSR has become an effective energy-saving and environmental protection technology. Foreign scholars, Kelso and Walker C A, have made a comprehensive elaboration on the mechanism of low frequency VSR [2-4]. The domestic professor Song Tianmin explained the mechanism of VSR by experiment from the angle of energy transformation in dislocation motion [5]. But these researches are mainly focused on the traditional low frequency VSR, whose excitation frequency is less than $200 \mathrm{~Hz}$, which is seldom involved in high frequency VSR of complex thin-walled workpieces with high natural frequencies.

Based on the theoretical study of the mechanism of high frequency VSR, aiming at the key parts of aerospace equipment, a modeling and simulation technology of high frequency VSR for complex thin-walled workpiece is proposed, through modal analysis and harmonic response analysis of finite element model of workpiece, the maximum dynamic stress of structure is calculated, the relationship curve between the maximum dynamic stress of structure and excitation frequency and amplitude of excitation force is established, and the high frequency VSR parameters are determined, which can be used to guide the practical engineering application.

\section{Mechanism of high frequency VSR}

According to lattice dynamics, the relationship between crystal particles in the crystal is regarded as a simple mass spring system, as shown in Figure 1, the motion equation of material particles with multiple degrees of freedom damped vibration system can be expressed as:

$$
M \ddot{x}+C \dot{x}+K x=F(t)
$$

In the formula (1), $M$ is the mass matrix, $C$ is the damping matrix, $K$ is the stiffness matrix, and $F(t)$ is the force vector.

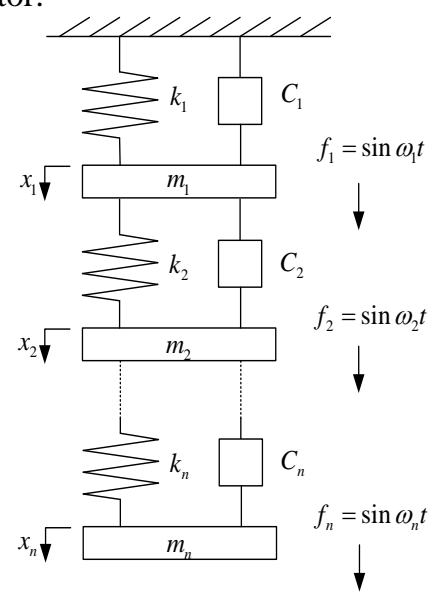

Figure 1. Multi degree of freedom damping vibration system diagram. 
According to the modal analysis theory, the displacement admittance ( frequency response function ) [6] of any mass element $m_{l}$ under the excitation of mass element $m_{p}$ can be expressed as:

$$
H_{l p}(\omega)=\sum_{i=1}^{N} \frac{\varphi_{l i} \varphi_{p i}}{K_{i}-\omega^{2} M_{i}+j \omega C_{i}}
$$

In the formula (2), $K_{i} 、 M_{i} 、 C_{i}$ are the i-th order damped modal stiffness, modal mass and modal damping. $\varphi_{l i} 、 \varphi_{p i}$ are the i-th order damped natural mode coefficient of $m_{l} 、 m_{p}$.

As can be seen from equation (2), for a system with multiple degree of freedom and damping, that system has multiple natural frequency, when the excitation force frequency is $\omega=\sqrt{1-2 \xi_{i}^{2}} \omega_{n i}$ ( $\xi_{i}$ is damping ratio, and $\xi_{i}=C_{i} /\left(2 \sqrt{M_{i} K_{i}}\right) ; \omega_{n i}$ is the i-th order natural frequency of the undamped mode, and $\left.\omega_{n i}=\sqrt{K_{i} / M_{i}}\right), m_{l}$ has the largest response and generate resonance. Figure 2 is a displacement frequency response diagram of multi-degree-of-freedom damped vibration system.

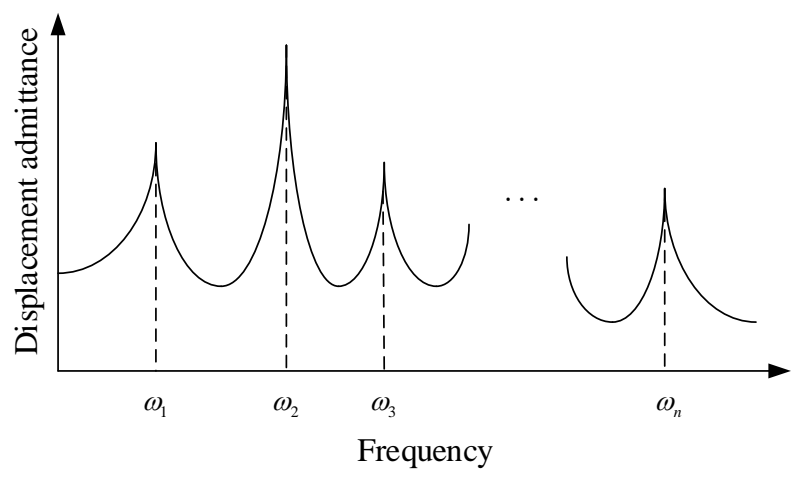

Figure 2. Displacement admittance graph of a multi degree of freedom damping vibration system.

When a high frequency vibration is applied to the crystal, the crystal system can be seen as a mass spring vibration system with multiple degrees of freedom. The system has multiple resonance frequencies, which are high because of the small mass of crystal particles and the large spring stiffness. However, under the condition of external high frequency excitation, when the external vibration frequency is consistent with a certain order resonance frequency of the system, the crystal grains in the crystal will move violently, so that the high-energy atoms in a stable state in the crystal material can obtain enough energy, thus getting rid of the bondage of the surrounding atoms and quickly returning to the original low-energy stable position. For crystal materials, dislocations and internal stresses disappear, so that we can eliminate the residual stress in the material.

The mechanism of common low frequency VSR is to cause dislocation proliferation, accumulation and entanglement in the material. Different from the mechanism of low frequency VSR. The mechanism of high frequency vibration aging is to make the dislocation density decrease or even disappear, which makes the material performance closer to the original stress free state. The residual stress distribution in the component is more uniform than the low frequency VSR, and the dimensional stability of the component is also better. At the same time, because of the high frequency excitation, the macroscopic deformation of the specimen is very small, so it is not easy to cause the fatigue damage of the specimen, which will lay the foundation for the popularization and application of the technology.

\section{Numerical simulation analysis of high frequency VSR of rotating frame}

\subsection{Establishment of finite element model of rotating frame}

The rotating frame is the main supporting structure of an aerospace equipment servo mechanism inner frame combination, which belongs to a typical complex thinwall workpiece, as shown in Figure 3, and its material is ZL114a. In order to facilitate the subsequent analysis of the structure and reduce the calculation, the key structure of the rotating frame is extracted on the basis of not affecting the simulation results, only the main features are retained, the parts are simplified and the structures such as guide angle, guide edge, fillet and reinforcement are removed, as shown in Figure 4.

The rotating frame material is ZL114A, and the material mechanics performance parameters, such as yield limit, modulus of elasticity, Poisson's ratio and density, are required to determine in the stress field analysis process. According to the manual of metal materials, the physical and mechanical properties of ZL114A material below $4000^{\circ} \mathrm{C}$ were found. The density was $2850 \mathrm{~kg} / \mathrm{m}^{3}$, the elastic modulus was $71 \mathrm{GPa}$, and the Poisson's ratio was 0.34 . When the initial residual stress is applied to the workpiece, the expansion coefficient of the temperature field analysis is $2.38 \mathrm{e}-5\left(1 /{ }^{\circ} \mathrm{C}\right)$.

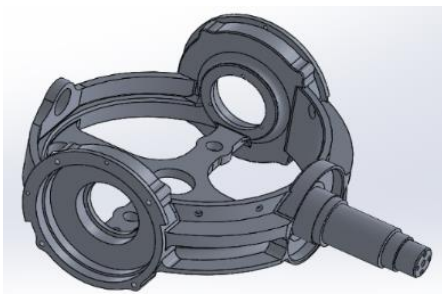

Figure 3. Original model.

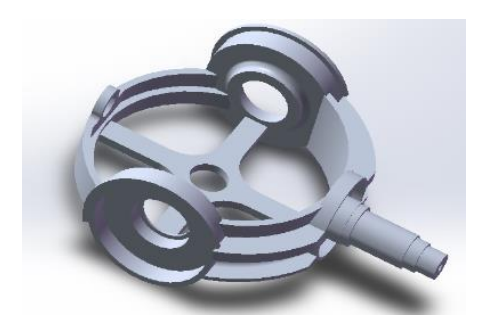

Figure 4. Simplified model. 
After a series of pre-processing such as simplifying the rotating frame model, defining the performance parameters and so on, the model is meshed and the finite element model is established. Figure 5 is the finite element model of a rotating frame.

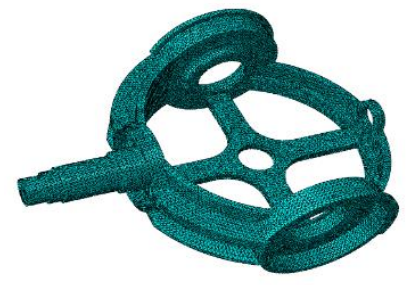

Figure 5. Finite element model.

\subsection{Modal analysis technology of rotating frame}

\subsubsection{Free mode analysis}

First of all, the free modal analysis of the rotating frame is carried out to get its natural mode, and the reasonable vibration mode is selected as the initial mode of high frequency vibration aging treatment, and the excitation point, support point and excitation frequency are determined according to the vibration mode.

Based on the finite element model established above, the frequency extraction analysis step is created, and the free modal analysis of the workpiece is carried out. The resonant frequencies and mode shapes of the first eight orders of the natural frequencies of the workpiece are extracted, and the corresponding mode shapes are described. The results of free mode analysis are shown in table 1 .

Table 1. Free mode analysis results.

\begin{tabular}{|c|c|c|}
\hline Order & $\begin{array}{c}\text { Natural } \\
\text { frequency } \\
(\mathrm{Hz})\end{array}$ & Mode description \\
\hline 1 & 0 & First order rigid body displacement \\
2 & 0 & Second order rigid body displacement \\
3 & 0 & Third order rigid body displacement \\
4 & $1.70 \mathrm{E}-03$ & Fourth order bending \\
5 & $2.60 \mathrm{E}-03$ & Fifth order bending \\
6 & $3.10 \mathrm{E}-03$ & Sixth order bending \\
7 & 1151.7 & Seventh order bending \\
8 & 1511.2 & Eighth order bending \\
\hline
\end{tabular}

Figure 6 is the free mode analysis nephogram of first to eighth order of a three-dimensional model of the rotating frame workpiece. It can be seen from the figure that the first three natural frequencies are all zero, corresponding to the rigid body displacement of the workpiece in three degrees of freedom. The mode shapes of the fourth, fifth and sixth order modes show a small bending state, and the natural frequency is very small, which is close to the displacement of the rigid body, so it is not suitable for VSR. In the seventh order modal shape, the displacement deformation near the circular hole in the middle of the workpiece is zero, and the four end parts of the workpiece are relatively large in displacement deformation. Therefore, the middle part of the workpiece with zero displacement deformation can be selected as a supporting point, a three-point support method is adopted, and the supporting points are arranged near the circular hole in the middle part, and the excitation point is selected at the right end part of the workpiece with relatively large displacement deformation. The eighth order modal shape is complex, and the vibration mode is ignored here.
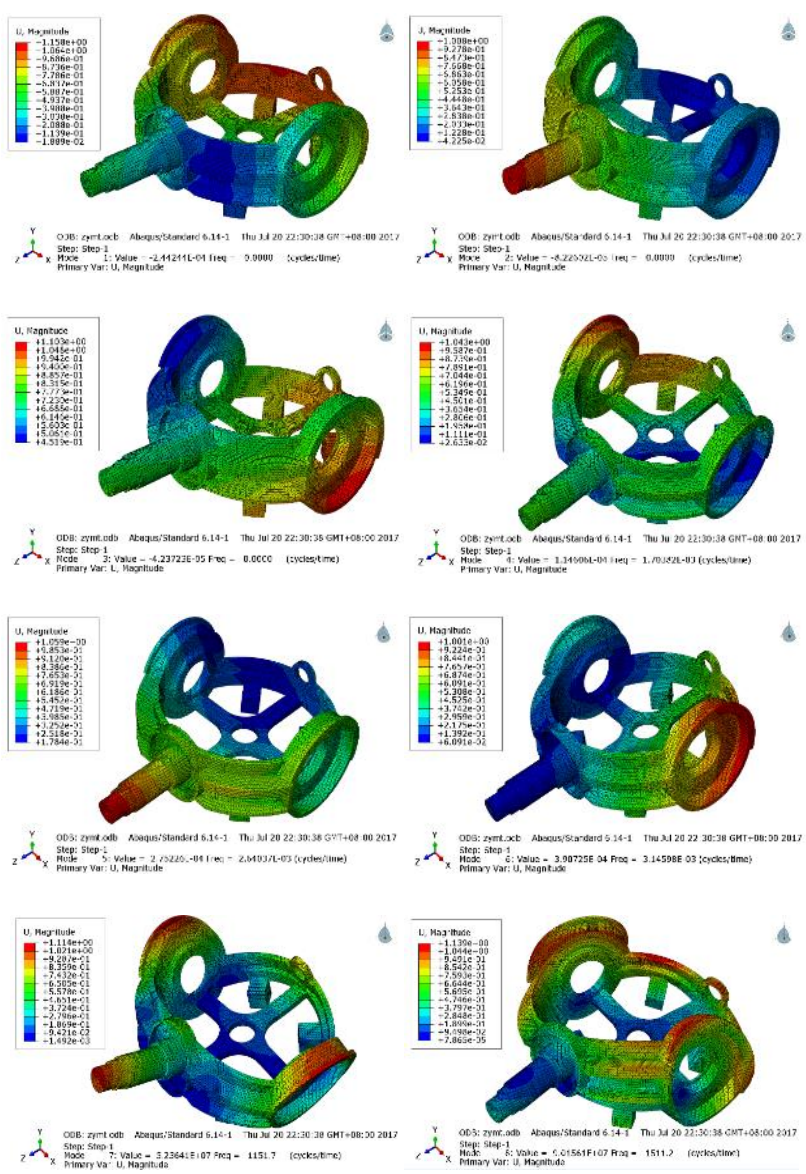

Figure 6. One to eight order free modal analysis nephogram of rotating frame.

The analysis shows that the bending vibration mode of the seventh order is selected as the initial vibration mode of the high frequency VSR, the natural frequency value is $1151.7 \mathrm{~Hz}$, three-point support is adopted, the supporting point of the VSR excitation is arranged in three directions of zero displacement near the circular hole. The larger displacement value, that is, the right end of the workpiece is selected as the excitation point of the high frequency VSR.

\subsubsection{Constrained mode analysis}

Next, constraint modal analysis of rotating frame is carried out. The simulation of the constrained modal analysis continues to follow the finite element analysis model of free mode analysis, and select the constraint points according to its seventh order mode, specify the number of modes to be analyzed, add linear boundary conditions. Then, analyze and solve the inherent 
frequency and corresponding mode of vibration, and enter the post processing to look at the analysis results.

On the basis of the free mode analysis, the seventh order mode of free vibration is taken as the prototype. According to the actual conditions of the experiment, the middle part of the workpiece is selected as the supporting point, and the three point support is adopted to add the three full degree of freedom constraint boundary conditions in the three directions of the middle hole of the model, and the constrained modal analysis of the rotating frame is carried out. The natural frequencies and modes of the first eight orders of the constrained modal analysis of the rotating frame are extracted, and the corresponding mode shapes are described, as shown in table 2 .

Table 2. Constrained mode analysis results.

\begin{tabular}{|c|c|c|}
\hline Order & $\begin{array}{c}\text { Natural } \\
\text { frequency } \\
(\mathrm{Hz})\end{array}$ & Mode description \\
\hline 1 & 348.95 & First order bending \\
2 & 594.58 & Second order bending \\
3 & 914.94 & Third order bending \\
4 & 929.57 & Fourth order bending \\
5 & 1182.4 & Fifth order bending \\
6 & 1573 & Sixth order bending \\
7 & 2295.4 & Seventh order bending \\
8 & 2404.4 & Eighth order bending \\
\hline
\end{tabular}

The first eight natural frequencies and mode shapes are still extracted, and the fifth order mode is selected with a natural frequency of $1182.4 \mathrm{~Hz}$. The vibration frequency is selected range from $1120 \mathrm{~Hz}$ to $1180 \mathrm{~Hz}$ by equation (3) [7].

$$
\omega=\omega_{1}+1 / 3\left(\omega_{0}-\omega_{1}\right) \sim \omega_{1}+2 / 3\left(\omega_{0}-\omega_{1}\right)
$$

In the formula (3): $\omega_{0}-\omega_{1}=\zeta \omega_{0}$, Where $\zeta$ represent that damping ratio of the material.

\subsection{Harmonic response analysis technology of rotating frame}

The finite element analysis model is consistent with the modal analysis process, and harmonic response analysis is carried out by using the same grid division method. This paper mainly introduces the following four problems that should be solved in harmonic response analysis.

(a) Determine the value of the exciting force

The strength limit, yield limit and fatigue limit of cast aluminum alloy $114 \mathrm{~A}$ can be known from the metal material handbook. As shown below:

$$
\begin{gathered}
\sigma_{b}=360 M P a \\
\sigma_{s}=325 M P a \\
\sigma_{-1}=\sigma_{b} / 3
\end{gathered}
$$

According to the standard formula of excitation force (4), the value of exciting force can be calculated.

$$
\sigma_{s}-\sigma_{r}<\sigma_{d}<\sigma_{-1}
$$

According to the calculation formula of excitation force (4), the range of dynamic stress can be obtained. (b) Determine the excitation frequency

According to the results of free modal analysis and constrained modal analysis, the range of excitation frequency is determined. It is known from the previous article the range of excitation frequency is $1120 \mathrm{~Hz}<\mathrm{F}_{0}<$ $1200 \mathrm{~Hz}$.

(c) Applied residual stress field

In the harmonic analysis of the rotating frame, the residual stress field in the process of the material should be taken into consideration. Therefore, the initial residual stress should be applied to the rotating frame work. The residual stress field of the workpiece is simulated by induction hardening. The initial temperature field is defined as $20{ }^{\circ} \mathrm{C}$ In the analysis step, the temperature of the hardened zone is raised to a certain temperature of $\mathrm{T}_{\text {high }}$, and the rest temperature remains $20{ }^{\circ} \mathrm{C}$. This temperature difference causes compressive stress in the high temperature region, which is equivalent to the simulated residual compressive stress. The maximum residual compressive stress of the workpiece can be obtained from the analysis of the residual stress field simulated by $282 \mathrm{MPa}$.

(d) Harmonic response analysis

Different excitation force amplitudes $\mathrm{F}_{0}$ are taken as $20 \mathrm{~N}, 40 \mathrm{~N}, 60 \mathrm{~N}, 80 \mathrm{~N}, 100 \mathrm{~N}$ for analysis and solution in the same frequency range $1120 \mathrm{~Hz}<\mathrm{F}_{0}<1200 \mathrm{~Hz}$, and the results are plotted as a graph of the relationship between the maximum stress value and the excitation frequency of the rotating frame at different excitation force amplitudes as shown in Figure 7.

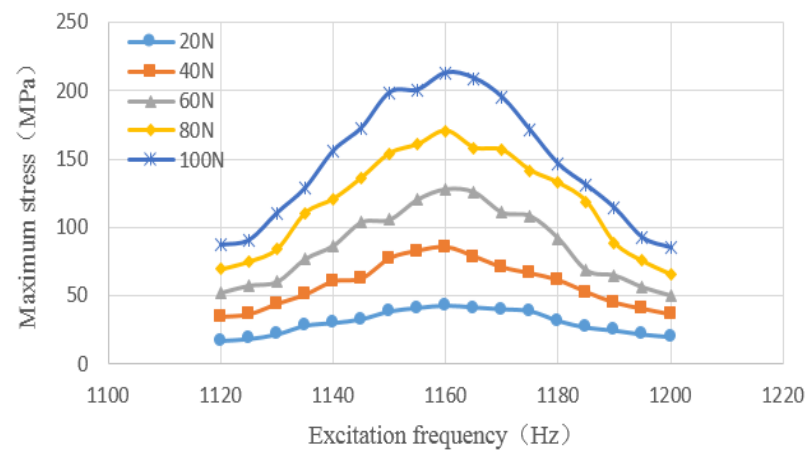

Figure 7. Frequency-maximum stress curve under different excitation force amplitudes.

As can be seen from that graph of frequency and maximum stress in Figure 7, under the action of different excitation force amplitudes, the rotary frame workpiece can generate the maximum equivalent stress value at the excitation frequency of $1160 \mathrm{~Hz}$. Its value reflects the effect of vibration stress relief to eliminate residual stress, so the excitation frequency can be chosen as $1160 \mathrm{~Hz}$. The relation between the exciting force and the maximum dynamic stress under the excitation frequency is shown in table 3.

The relation curve between the maximum dynamic stress and the exciting force of the rotating frame structure at $1160 \mathrm{~Hz}$ is made, as shown in Figure 8. According to the mechanism of VSR, the sum of dynamic stress and residual stress should be greater than the yield limit of the vibrating material, and the 
dynamic stress is less than the fatigue limit of the material. Therefore according to the dynamic stress standard calculation formula $\sigma_{\mathrm{s}}-\sigma_{\mathrm{r}}<\sigma_{\mathrm{d}}<\sigma_{-1}$, the range of dynamic stress that can be obtained is $43 \mathrm{MPa}<\sigma_{\mathrm{d}}<$ $120 \mathrm{MPa}$. According to the relationship between the maximum dynamic stress and the excitation force shown in Figure 8, we can obtain the amplitude range of the required harmonic excitation force is $21.2 \mathrm{~N}<\mathrm{F}_{0}<$ $56 \mathrm{~N}$, and the $\mathrm{F}_{0}=25 \mathrm{~N}$ is preferable for experiment.

Table 3. The relationship between the exciting force and the maximum dynamic stress when frequency is $1160 \mathrm{~Hz}$.

\begin{tabular}{|c|c|c|c|c|c|}
\hline $\begin{array}{c}\text { Exciting } \\
\text { force/N }\end{array}$ & 20 & 40 & 60 & 80 & 100 \\
\hline $\begin{array}{c}\text { Maximum } \\
\text { dynamic } \\
\text { stress/Mpa }\end{array}$ & 42.6 & 85.2 & 127.8 & 170.4 & 213 \\
\hline
\end{tabular}

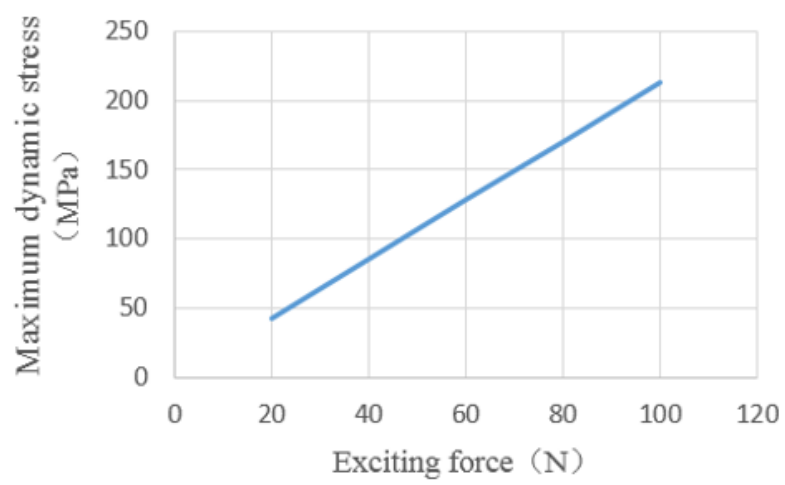

Figure 8. Curve of excitation force and maximum dynamic stress at excitation frequency of $1160 \mathrm{~Hz}$.

\section{Analysis of high frequency VSR simulation results of rotating frame}

The high frequency excitation simulation is carried out at $25 \mathrm{~N}$ excitation force and $1160 \mathrm{~Hz}$ excitation frequency, the stress distribution of the workpiece before and after excitation is shown in Figure 9. Ten nodes with obvious structural characteristics are selected, and the residual stress values before and after high frequency VSR treatment ( harmonic response analysis ) are recorded respectively, the results are plotted as a graph as shown in Figure 10.

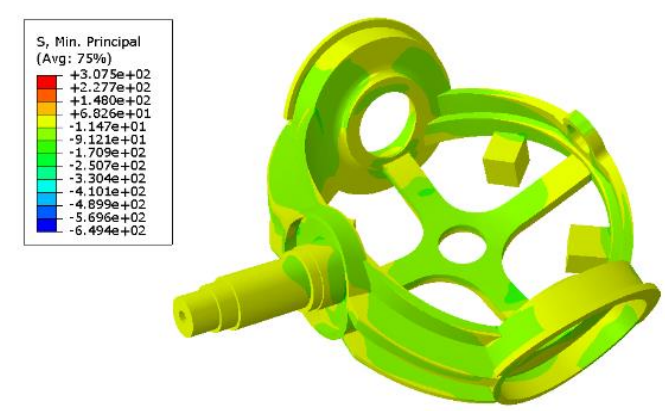

(a) Before harmonic response analysis.

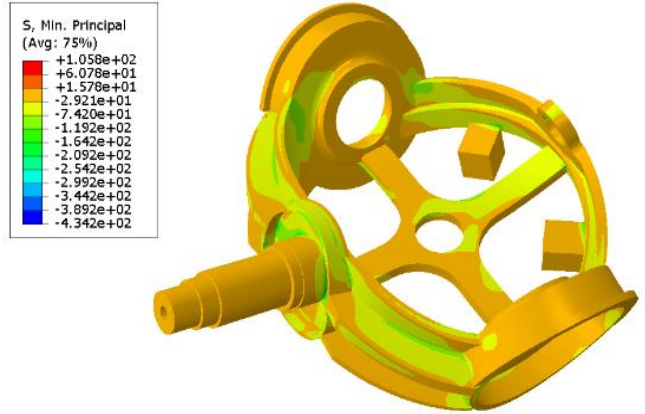

(b) After harmonic response analysis.

Figure 9. Stress distribution of workpiece before and after harmonic response analysis.

Comparing and analyzing the stress data before and after the high frequency VSR treatment in Figure 10, the excitation force has different influence on the residual stress field in different regions of the rotating frame. Where the initial residual stress value is larger, the reduction of residual stress is larger, and the homogenization degree is also higher.

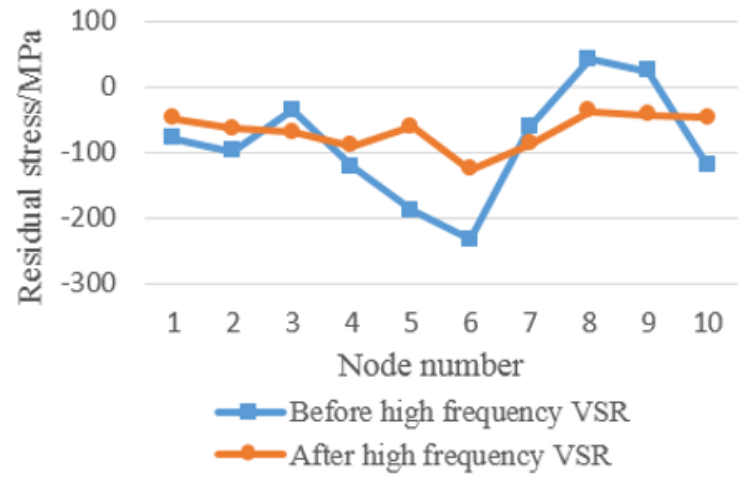

Figure 10. Stress change before and after high frequency VSR simulation analysis.

\section{Conclusion}

In this paper, the mechanism of high frequency VSR is studied by microdynamic theory, and the relationship between material particles in the specimen is considered as a damping vibration system with multiple degrees of freedom. Under the condition of vibration excitation, when the external vibration frequency is consistent with a certain resonance frequency, the particles will move violently and quickly return to the original stable equilibrium position, so as to reduce and homogenize the internal residual stress of the material, and the material performance is closer to the original stress-free state.

Taking the analysis of typical complex thin-walled workpiece rotating frame as an example, the finite element model is established, and the high frequency VSR parameters are determined by combining modal analysis and harmonic response analysis technology. The simulation results are analyzed to verify the effect of reducing and homogenizing residual stress, which provides a theoretical basis for the optimization of high frequency VSR excitation parameters. Further technical development and implementation will be carried out in 
combination with the actual high frequency VSR experiment.

\section{References}

1. Zhang Yong, Chin. J. Mech. Eng 13(19) pp 16401642 (2002)

2. C.A. Walker, A.J. Waddell, D.J. Johnston, Proc. Inst. Mech. Eng, Part E: J. Proc. Mech. Eng 209(1) pp 51-58 (1995)

3. G. Gnirss, Weld. World 26(11) pp 284-291 (1988)
4. A.S. Munsi, A.J. Waddell, C.A. Walker, J. Stra. Anal. Eng. Des 36(5) pp 453-464 (2001)

5. Song Tianmin, Research on the mechanism of VSR (J. Jilin. U: Techno. Ed (1) pp 53-56, 1995)

6. Chen Yaodong, Mechanical vibration (Hangzhou: Zhejiang University Press) (1988)

7. Li Quanhua, Heat treatment technology 400 questions and analysis (Beijing: Machinery Industry Press) pp 552-553 (2002) 